\title{
HSV-1 thymidine kinase gene therapy for colorectal adenocarcinoma-derived peritoneal carcinomatosis
}

\author{
C Lechanteur ${ }^{1}$, F Princen ${ }^{1}$, S Lo Bue ${ }^{2}$, B Detroz ${ }^{2}$, G Fillet ${ }^{1}$, J Gielen ${ }^{1}$, V Bours ${ }^{1}$ and M-P Merville ${ }^{1}$ \\ ${ }^{1}$ Laboratory of Medical Oncology and Medical Chemistry and ${ }^{2}$ Department of Abdominal Surgery, University of Liège, Belgium
}

Peritoneal carcinomatosis is a common clinical situation which, in most cases, cannot be eradicated by surgery or chemotherapy. The feasibility of an HSV-TK-based suicide gene therapy for peritoneal carcinomatosis induced by DHD/K12 colon carcinoma cells was investigated. DHD/K12 cells stably expressing the tk gene were killed in vitro in the presence of low concentrations of ganciclovir; they exhibited a 'bystander effect' when mixed with TKnegative cells. BD-IX rats injected intraperitoneally, either directly or after surgical peritoneal irritations, with $\mathrm{DHD} / \mathrm{K} 12$ cells developed peritoneal carcinomatosis within 2 weeks. Ganciclovir treatment of animals injected with $D H D / K 12$ TK cells allowed a significant reduction of the tumor volume as well as a prolonged survival. Of these animals 35-40\% showed a long-term disease-free survival after ganciclovir therapy. Residual or relapsing tumors could be explained by a low expression of the transgene as demonstrated by RT-PCR.

Keywords: gene therapy; thymidine kinase; peritoneal carcinomatosis; colorectal cancer

\section{Introduction}

Peritoneal dissemination is a common clinical situation in oncology which usually results from ovarian or digestive primary neoplasms. ${ }^{1}$ Surgical resection of extensive peritoneal carcinomatosis is impossible and most of these cancers, including colorectal carcinomas, are poorly reactive to systemic chemotherapy. Intraperitoneal delivery of cytotoxic drugs which allows high local concentration could lead to clinical benefits in some circumstances. ${ }^{1,2}$

Suicide gene therapy consists of the intracellular delivery of a gene coding for an enzyme which transforms a prodrug into a cytotoxic product. ${ }^{3}$ Thymidine kinase from the herpes simplex virus type I (HSV-TK) is the most commonly used enzyme. It phosphorylates the nucleoside analogue ganciclovir (GCV) and allows its further transformation, after subsequent phosphorylation by cellular kinases, into cytotoxic ganciclovir-tri-phosphate, which inhibits cellular DNA polymerase. ${ }^{4-6}$ Numerous in vitro and in vivo studies have demonstrated that tumor cells expressing HSV-TK are specifically killed by the antiviral drug GCV. ${ }^{7-11}$ Moreover, untransduced adjacent cells are also sensitive to the GCV cytotoxic effect ('bystander effect'). ${ }^{10,12-14}$ For in vivo transduction, the suicide gene is, in most cases, inserted into a viral vector which is directly injected at the tumor site. ${ }^{14-19}$ Several clinical studies currently underway are based on a similar approach. ${ }^{3}$ Alternatively, several investigators have envisaged the delivery of the gene therapy to the

Correspondence: $V$ Bours, Medical Oncology, CHU B35, University of Liège, Sart-Tilman, 4000 Liège, Belgium

The two first authors contributed equally

Received 23 May 1997; accepted 4 July 1997 anatomical cavities in which the cancer has disseminated. ${ }^{12,20-22}$

The present report investigates the feasibility of an HSV $t k$-based suicide gene therapy in a model of peritoneal carcinomatosis induced by DHD/K12 colorectal adenocarcinoma cells in BD-IX syngeneic rats. Following intraperitoneal injection of DHD/K12 cells transduced with the HSVtk gene, treatment with GCV drastically reduces the tumor burden and statistically improves survival.

\section{Results}

\section{Ganciclovir cytotoxicity for HSV-TK-expressing DHD/K12 cells}

Rat colorectal adenocarcinoma DHD/K12 cells were transduced in vitro with a retroviral vector containing the thymidine kinase gene from HSV-1 under the control of the SV40 promoter (DHD/K12-TK). PCR on genomic DNA and RT-PCR on total RNA confirmed that the HSV tk gene was integrated and expressed in the infected DHD/K12-TK cells (data not shown and Figure 1).

The cytotoxicity of increasing concentrations (0.1-5 $\mu \mathrm{m})$ of the prodrug GCV was then tested on the DHD/K12-TK cells. Figure 2 shows that GCV concentrations as low as $0.5 \mu \mathrm{m}$ killed the majority of these cells while untransfected DHD/K12 cells were resistant to much higher concentrations. The $\mathrm{IC}_{50}$ is $0.4 \mu \mathrm{m}$ for DHD/K12-TK cells and $82 \mu \mathrm{m}$ for untransfected cells (data not shown).

\section{Bystander effect between DHD/K12-TK and DHD/K-12} cells

In order to test whether a bystander effect could be observed, various proportions of untransfected and 


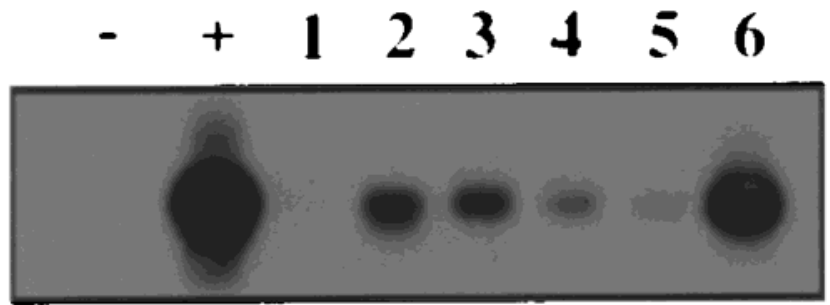

Figure 1 Tumor nodes at relapse faintly express the TK transgene. Tumor nodes at death from relapse were analyzed by RT-PCR for TK expression followed by Southern blotting. Lane (-) shows the negative control (PCR performed on water) and lane (+) shows the RT-PCR performed on the DHD/K12-TK cells. $4 \mu \mathrm{l}$ of the RT-PCR reaction were loaded on lane + while $20 \mu l$ were loaded on all the other lanes. Lanes 1-3 show the expression of the TK $m R N A$ in relapsing tumor nodes from animals injected with $20 \%(1), 50 \%$ (2) and $100 \%$ (3) DHD/K12-TK cells after peritoneal irritations, respectively. Lanes 4-6 show the expression of the TK gene in relapsing tumor nodes from animals directly injected with $20 \%$ (4), $50 \%$ (5) and $100 \%$ (6) DHD/K12-TK cells, repectively. A faint band is visible in lane 1 after longer exposures.

transfected DHD/K12 cells were cocultured and challenged for 7 days with GCV at a concentration that kills $100 \%$ of DHD/K12-TK cells and does not affect untransfected cells $(20 \mu \mathrm{m})$ (Figure 3). Under these experimental conditions, the presence of $25 \%$ of DHD/K12-TK cells was sufficient to induce a cytotoxic effect which killed $89 \%$ of the cells. This experiment also confirmed that the survival of untransfected DHD/K12 cells was unaffected by the GCV treatment.

\section{In vivo model for suicide gene therapy of peritoneal carcinomatosis}

A model of peritoneal carcinomatosis induced by DHD/K12 cells in syngeneic BD-IX rats was chosen to test the feasibility of an HSVtk-based gene therapy approach. BD-IX rats were injected intraperitoneally at day 0 with $10^{6} \mathrm{DHD} / \mathrm{K} 12-\mathrm{TK}$ cells or with $10^{6}$ untransduced DHD/K12 cells. These animals developed peritoneal tumor nodes on the epiploon within 10 days and

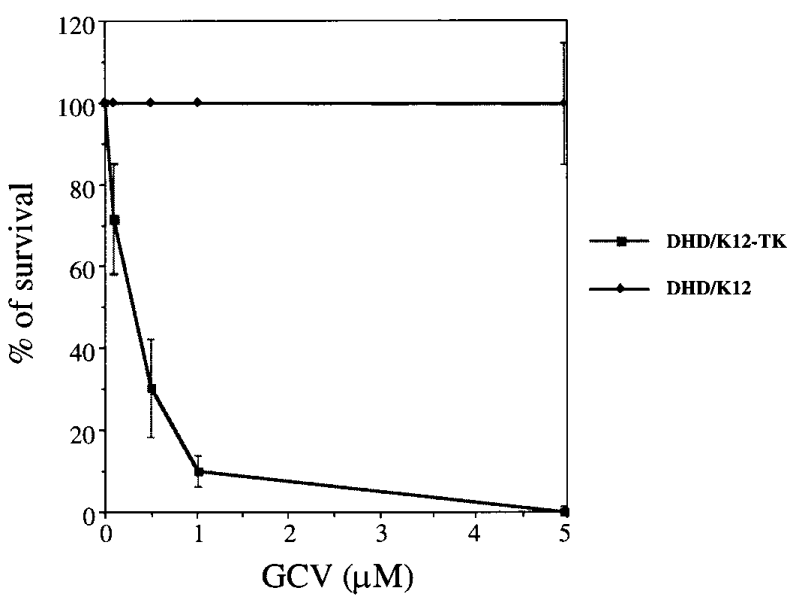

Figure 2 Cytotoxic effect of ganciclovir on DHD/K12-TK cells in vitro. Stably transfected DHD/K12-TK cells and untransfected DHD/K12 cells were treated in vitro for 7 days with increasing concentrations of GCV. Cell viability was estimated with the WST-1 test. The concentrations of GCV which killed $50 \%$ of the cells $\left(I_{50}\right.$ ) were $0.4 \mu \mathrm{m}$ for DHD/K12-TK cells and $82 \mu \mathrm{m}$ for untransfected cells, respectively. untreated controls died of extensive peritoneal dissemination between days 50 and 60 (Figures 4 and 5).

Two rats were injected at day 0 with untransduced DHD/K12 cells while groups of two animals were injected with DHD/K12-TK cells. These last groups injected with DHD/K12-TK cells were then treated intraperitoneally with increasing doses of GCV for 5 days from day 14 to test the efficiency and safety of the method. The animals were killed at day 21 and the reduction of the tumor volume was assessed by direct abdominal examination. We observed a major decrease in the number and volume of tumor nodes in the animals injected with DHD/K12-TK cells and treated with GCV as compared with animals treated with HBSS or injected with untransfected cells (Figure 4, compare $\mathrm{c}$ with a and b). Longer treatments were also investigated and, although it is premature to draw any definitive conclusion with such a low number of animals and in the absence of survival data, direct abdominal evaluation of dead animals did not allow us to observe any obvious differences in efficacy with treatments prolonged up to 14 days. Moreover, we did not observe any obvious toxicity of GCV at doses ranging from $35 \mathrm{mg} / \mathrm{kg}$ once a day to $150 \mathrm{mg} / \mathrm{kg}$ once a day or $75 \mathrm{mg} / \mathrm{kg}$ twice a day (data not shown). The dose of GCV for further treatment was thus fixed at $150 \mathrm{mg} / \mathrm{kg}$ injected intraperitoneally once a day for 5 days starting at day 14. Similar or higher doses had previously been administered by others without any detectable toxicity. ${ }^{14}$

Animals were then injected with various proportions of DHD/K12-TK and untransfected DHD/K12 cells and treated with GCV using the same protocol. Eight rats were analyzed for each proportion of transduced/ untransduced cells. The eight rats injected with $0 \%$ DHD/K12-TK cells died between days 50 and 56 (median survival: 53 days) (Figure 5) as did the control animals treated with the buffer alone (data not shown). Animals injected with $20 \%$ of DHD/K12-TK cells and treated with GCV showed a significantly prolonged survival as compared with the animals injected with $0 \% \mathrm{DHD} / \mathrm{K} 12-\mathrm{TK}$

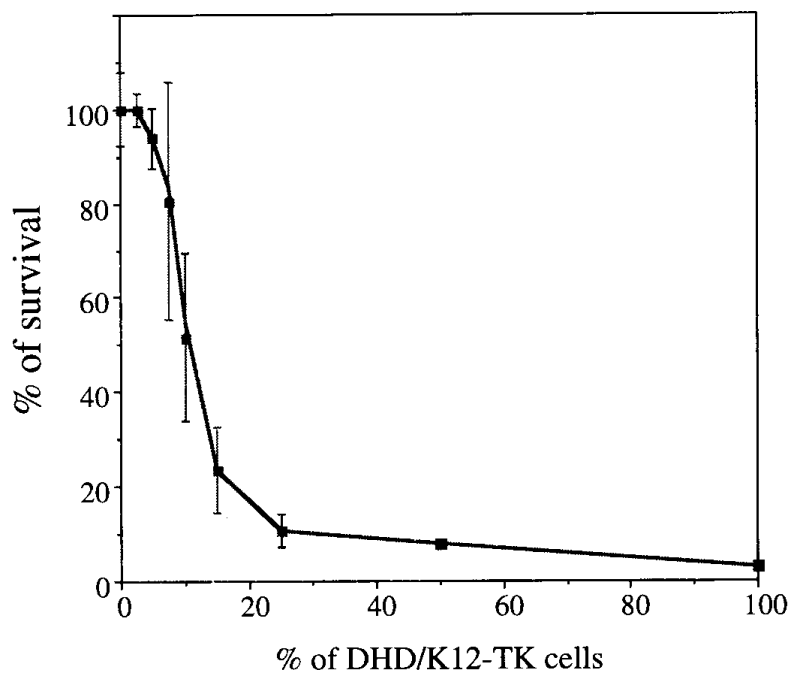

Figure 3 In vitro bystander effect between DHD/K12-TK and untransfected cells. DHD/K12-TK cells and untransfected cells were cocultured in various proportions as indicated. These cocultures were treated with $20 \mu \mathrm{m}$ of GCV for 7 days and cell viability was measured with the WST1 test. 
a

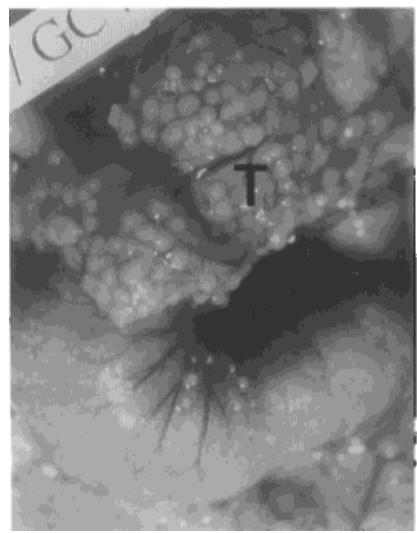

b

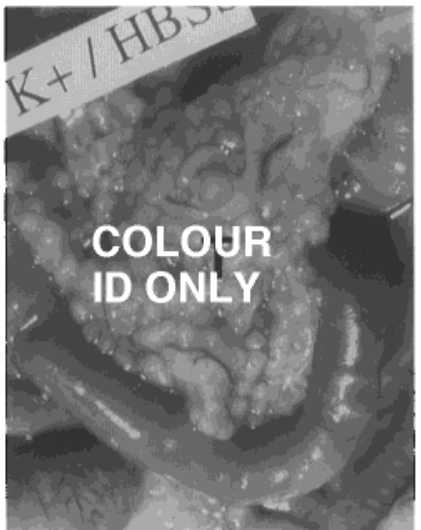

C

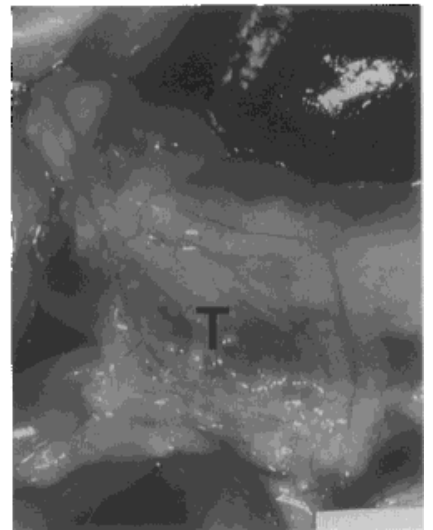

Figure 4 Ganciclovir treatment of rats injected with DHD/K12-TK cells. The figure shows the peritoneal cavity at day 21 of a rat injected with DHD/K12 untransfected cells and treated with GCV (a), a rat injected with DHD/K12-TK cells and treated with the HBSS buffer alone (b), and a rat injected with DHD/K12-TK cells and treated with GCV for 5 days at $150 \mathrm{mg} / \mathrm{kg}$ (c). Groups of two rats were injected with DHD/K12 or DHD/K12-TK cells and treated with GCV for 5 days at doses from $35 \mathrm{mg} / \mathrm{kg}$ once a day to $150 \mathrm{mg} / \mathrm{kg}$ once a day or $75 \mathrm{mg} / \mathrm{kg}$ twice a day. The animals were killed at day 21 and a representative picture is shown in the figure. The letter ' $T$ ' indicates the peritoneal tumor nodes.

cells (log-rank test: $P<0.01)$. Animals injected with $50 \%$ of DHD/K12-TK cells again showed a significantly prolonged survival following GCV treatment as compared with the $0 \% \mathrm{DHD} / \mathrm{K} 12-\mathrm{TK}$ group $(P<0.05)$. Indeed, in each of the $20 \%$ and $50 \%$ DHD/K12-TK groups, a small number of animals (12.5 and $25 \%$, respectively) showed a prolonged survival and were tumor-free at day 270 . Moreover, the rats injected with $100 \%$ DHD/K12-TK cells showed a significant increase in survival after a single 5day treatment with GCV $(P<0.01)$ (Figure 5). In this last group, three animals $(37.5 \%)$ showed a long survival and were free of tumors at day 270.

Surgical wounds favor the implantation of tumor cells in the peritoneal cavity and the development of tumor nodes. To reproduce the surgical procedure, rats were laparotomized and a dissection of the sigmoid area was performed without any intestinal resection. $10^{6}$ $\mathrm{DHD} / \mathrm{K} 12$ cells were then directly injected locally. Under these conditions, large tumor nodes quickly developed at the sites of peritoneal irritations (data not shown). We injected various proportions of DHD/K12-TK and DHD/K12 untransfected cells after surgical peritoneal irritations and treated these animals with intraperitoneal injections of GCV for 5 days starting at day 14. Rats injected with $100 \%$ DHD/K12-TK cells and treated with GCV showed a major reduction of their tumor nodes as compared with control animals treated with the buffer alone (data not shown). Animals injected with $0 \%$ DHD/K12-TK cells and treated with GCV or rats treated with the buffer alone died between days 53 and 63 (median survival: 56 days) (Figure 5 and data not shown). Animals injected with 20\% DHD/K12-TK cells and treated with GCV showed an increased survival as compared to animals injected with $0 \%$ DHD/K12-TK cells (log-rank test: $P<0.05$ ). The animals injected with $50 \%$ DHD/K12-TK cells did not survive longer than control animals, while animals injected with $100 \%$ DHD/K12-TK cells had a significantly longer survival $(P<0.0001)$. One animal from the $20 \%$ DHD/K12-TK cell group (12.5\%) and three animals from the $100 \%$ DHD/K12-TK cell group $(42.8 \%)$ were still alive and tumor-free at day 308 .

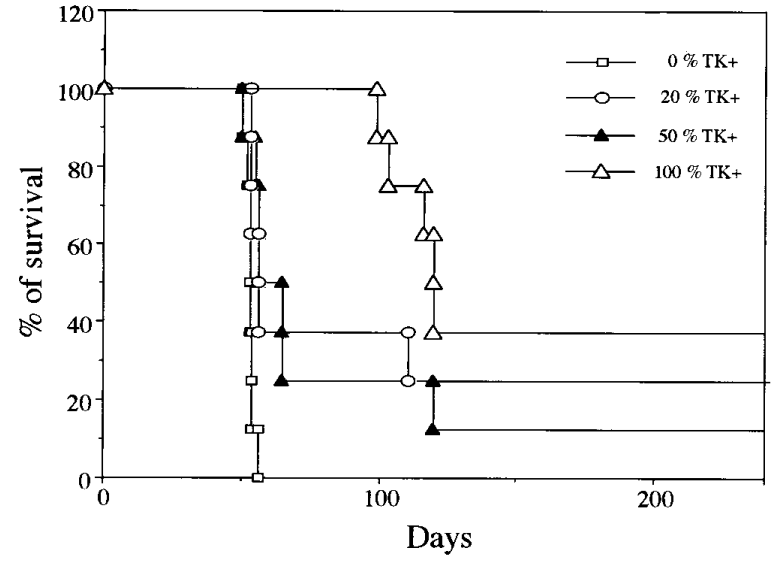

Figure 5 Survival of rats injected with DHD/K12-TK cells and treated with ganciclovir. For each group, eight animals were injected intraperitoneally with $10^{6} \mathrm{DHD} / \mathrm{K} 12$ cells containing $0 \%(\square), 20 \%(\mathrm{O}), 50 \%(\mathbf{\Delta})$ or $100 \% \mathrm{DHD} / \mathrm{K} 12-\mathrm{TK}$ cells $(\triangle)$, respectively. The rats were treated for 5 days starting at day 14 with GCV $150 \mathrm{mg} / \mathrm{kg}$ once a day (intraperitoneal injections). Survival curves for each group are shown. One rat from the $20 \% \mathrm{DHD} / \mathrm{K} 12-\mathrm{TK}$ group $(12.5 \%)$, two rats from the $50 \% \mathrm{DHD} / \mathrm{K} 12-$ TK group $(25 \%)$ and three rats from the $100 \%$ DHD/K12-TK group (37.5\%) were still alive and tumor-free at day 270.

\section{Low expression of the transgene in relapsing tumors}

Following a 5-day GCV treatment, a dramatic reduction of the tumor burden as well as a significant increase in the median survival was observed in all groups injected, either directly or after peritoneal irritations, with $100 \%$ DHD/K12-TK cells. However, only a minority of these animals showed a complete response with most relapsing and finally dying of tumor progression. The expression of HSV-TK in tumor nodes after relapse and death by tumor progression was investigated by RT-PCR followed by Southern blotting revealed by a TK probe (Figure 1). Tumor samples were collected from animals injected with 20,50 or $100 \%$ DHD/K12-TK cells after peritoneal irritation or not. Only a single tumor node from an animal injected with $100 \%$ DHD/K12-TK cells showed a 


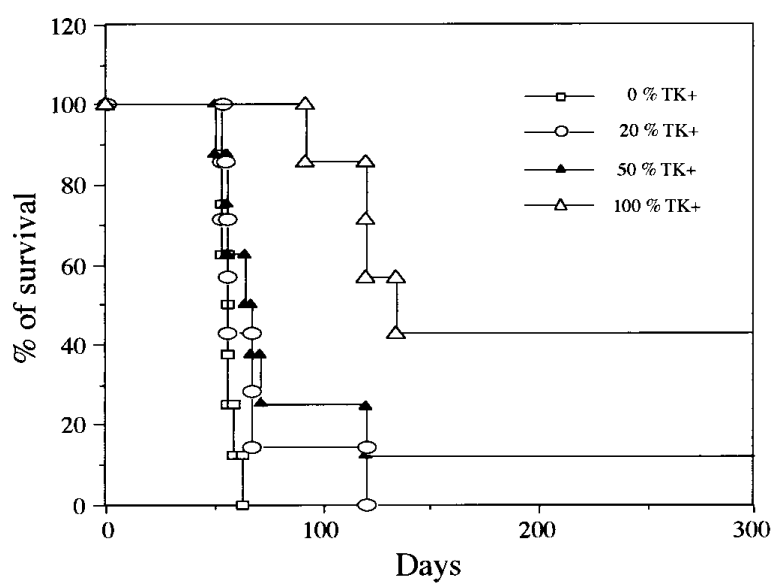

Figure 6 Survival of rats injected with DHD/K12-TK cells after peritoneal irritations. For each group, seven to eight animals were laparotomized for peritoneal irritations and injected locally with $10^{6} \mathrm{DHD} / \mathrm{K} 12$ cells containing $0 \%(\square), 20 \%(O), 50 \%(\mathbf{\Delta})$ or $100 \%$ DHD/K12-TK cells $(\triangle)$, respectively. The rats were treated for 5 days starting at day 14 with GCV $150 \mathrm{mg} / \mathrm{kg}$ once a day (intraperitoneal injections). Survival curves for each group are shown. One rat from the $20 \% \mathrm{DHD} / \mathrm{K} 12-\mathrm{TK}$ group $(12.5 \%)$, and three rats from the $100 \%$ DHD/K12-TK group (42.8\%) were still alive and tumor-free at day 308.

strong expression of the HSV-TK mRNA although this expression was much lower than in the original DHD/K12-TK cells (Figure 1, compare lanes + and 6; 4 $\mu \mathrm{l}$ of the RT-PCR reaction were loaded on lane + while $20 \mu \mathrm{l}$ were loaded on all the other lanes). All the other tumor nodes showed a weak but still detectable HSV-TK expression (lanes 1-5). Thus it is likely that relapsing tumor nodes still contained a low number of cells which can still express the $t k$ gene.

\section{Repeated ganciclovir courses}

To improve the efficiency of the therapy, rats injected with various proportions of DHD/K12-TK and untransduced $\mathrm{DHD} / \mathrm{K} 12$ cells were treated by repeated 5-day cycles of GCV (150 mg/kg, once daily). The first treatment was administered for 5 days at day 14 as in the experiment described above (Figure 5) and was followed by additional 5-day GCV cycles repeated every 4 weeks (Figure 7). The group injected with $20 \%$ DHD/K12-TK cells showed no significantly longer survival after repeated GCV cycles but the groups injected with 50 or $100 \%$ DHD/K12-TK cells showed a significant increase of survival (log-rank test: $P=0.015$ and $<0.01$, respectively) when compared with animals injected with untransduced cells only. However, repeated GCV treatments did not generate any survival advantage as compared with a single cycle (Figure 5). Again, one of five animals from the $50 \% \mathrm{DHD} / \mathrm{K} 12-\mathrm{TK}$ group and two of five animals from the $100 \%$ DHD/K12-TK group experienced a long survival and were alive and tumor-free at day 130.

\section{Discussion}

Peritoneal carcinomatosis is a common clinical situation which, in most cases, cannot be eradicated by surgery or chemotherapy. There is, therefore, an urgent need for novel therapeutic approaches. Intraperitoneal suicide gene delivery should make it possible to confine gene

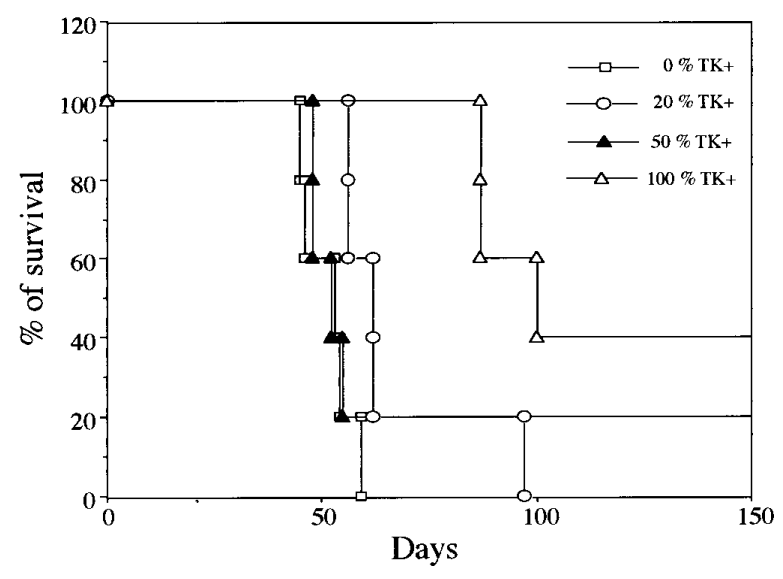

Figure 7 Survival of rats injected with DHD/K12-TK cells and treated with repeated cycles of ganciclovir. As described in Figure 5, animals were injected with $10^{6} \mathrm{DHD} / \mathrm{K} 12$ cells containing 0, 20, 50 or $100 \%$ DHD/K12-TK cells, respectively, and then treated with GCV. GCV was administered at $150 \mathrm{mg} / \mathrm{kg} / \mathrm{once}$ daily in 5-day cycles repeated every 4 weeks, starting at day 14. Five animals were treated in each group. One rat from the $50 \% \mathrm{DHD} / \mathrm{K} 12-\mathrm{TK}$ group $(20 \%)$ and two rats from the $100 \%$ DHD/K12-TK group (40\%) were still alive and tumor-free at day 150.

transduction to peritoneal cells and could therefore be suitable for such pathological conditions.

In this article, the feasibility of an HSVtk-mediated gene therapy for peritoneal carcinomatosis induced by colorectal adenocarcinoma cells has been investigated in a syngeneic immunocompetent animal model. BD-IX rats which had developed peritoneal carcinomatosis induced by DHD/K12 cells expressing the $t k$ gene showed a prolonged survival after GCV treatment when compared with animals injected with tumor cells which do not express the tk gene or with animals treated with the buffer alone. Although most animals relapsed and finally died of tumor progression, $35-40 \%$ of them demonstrated a long survival and remained tumor-free. These data thus indicated that a combined TK-GCV approach is efficient in this model and could be considered for the treatment of peritoneal carcinomatosis. Our observation supports that of several groups who have developed different animal models for gene therapy of peritoneal carcinomatosis. $12,20-22$

It is generally considered that, after in vivo gene transduction, only a small proportion of tumor cells captures the transgene. Therefore, the potential efficiency of an in vivo tk gene therapy relies on the 'bystander effect'. As demonstrated in this article, DHD/K12 colon cancer cells do exhibit a bystander effect in vitro. Consistent with this observation, the animals injected with 20 or $50 \%$ DHD/K12-TK cells mixed with untransfected DHD/K12 cells showed a significantly increased survival as compared with rats injected with DKD/K12 control cells. Indeed, in the 20 or $50 \% \mathrm{DHD} / \mathrm{K} 12-\mathrm{TK}$ groups, a few animals (10-20\%) showed a prolonged survival and remained apparently tumor-free. Several mechanisms can account for an in vivo bystander effect: a diffusion of the phosphorylated GCV from cell to cell through the gap junctions, the phagocytosis of apoptotic vesicles by adjacent cells, or an antitumor immune reaction.12,14,23-25 Therefore, we could conclude either that the GCV therapy allowed a complete eradication of DHD/K12 cells in these animals showing prolonged survivals or 
that a few animals developed an immune reaction which eliminated residual tumor cells. BD-IX rats are immunocompetent but DHD/K12 cells are considered to be poorly immunogenic and even immunosuppressive. ${ }^{26}$ It is however possible that the long-term survivors developed a specific antitumor immune reaction. Further experiments are needed in order to evaluate the immunity of these animals and to determine if immunomodulation could increase their survival rates.

RT-PCR performed on relapsing tumor nodes detected a weak $T K$ expression. It is thus likely that these tumors contained a large majority of cells that did not express the $t k$ gene mixed with a low number of transgeneexpressing cells. The failure of repeated GCV treatments suggests that the number of TK-expressing cells in these relapsing tumors is insufficient to generate a bystander effect and a tumor shrinkage. When in vivo transduction is performed, repeated injections of the transgene-containing vector will probably be required in order to maintain a significant number of cells expressing the $t k$ gene.

In this paper, cells were transduced in vitro before animal injections. Such an approach is not applicable in most clinical settings and experiments are in progress in our laboratory to develop appropriate techniques for in vivo gene delivery. Indeed, the model we describe here is suitable for the investigation of intraperitoneal delivery of suicide genes in immunocompetent animals.

\section{Materials and methods}

\section{Cell lines and in vitro treatment}

DHD/K12/Prob cells ${ }^{27}$ were cultured in DMEM-modified Dulbecco's medium (GIBCO BRL, Frederick, MD, USA) supplemented with $5 \%$ fetal bovine serum (FBS), $1 \%$ glutamine $200 \mathrm{~mm}, 1 \%$ Hepes $1 \mathrm{~m}, 1 \%$ arginine 0.55 $\mathrm{mm}$ and antibiotics. To test GCV cytotoxicity, $5 \times 10^{3}$ cells were incubated in $200 \mu \mathrm{l}$ of medium plus $15 \%$ FBS on $96-$ well plates. GCV (Cymevene; Syntex Laboratories, Palo Alto, CA, USA) was diluted in sterile water and added to the culture medium to reach the concentrations indicated in the figures. Cells were treated with GCV for 7 days and the medium + GCV was replaced at days 4 and 6 .

To test the bystander effect, DHD/K12-TK cells and untransfected cells were cocultured in various proportions $(0,2.5,5,7.5,10,15,25$ and $50 \%$ of DHD/K12TK cells, respectively). These cocultures were then challenged with $20 \mu \mathrm{m}$ of GCV for 7 days.

After 7 days of treatment, cell viability was tested with the WST-1 cell proliferation reagent as recommended by the manufacturer (Boehringer Mannheim, Mannheim, Germany).

\section{Retroviral vector and gene transduction}

The retroviral vector, derived from a murine retrovirus (MoMLV), was a generous gift from Dr C-M Calberg (Laboratory of Virology, University of Liège, Liège, Belgium). This vector contains the neomycin resistance gene driven by the first LTR and the HSV1tk gene driven by an SV40 promoter. The packaging cell line was the GP+EnvAm12 line. ${ }^{28}$

After titration on NIH3T3 cells, three dilutions of supernatant (final volume: $3 \mathrm{ml}$ ) from GP+EnvAm12 cells were placed on $7.5 \times 10^{5} \mathrm{DHD} / \mathrm{K} 12$ cells on a $100-\mathrm{mm}$ tissue culture dish in the presence of polybrene (8 $\mu \mathrm{g} / \mathrm{ml})$. After $150 \mathrm{~min}$ of incubation at $37^{\circ} \mathrm{C}, 7 \mathrm{ml}$ of fresh medium was added to the cells. Twenty-four hours later, the cells were diluted and selected for 2 weeks in the presence of G418 (active concentration: $500 \mu \mathrm{g} / \mathrm{ml}$ ).

\section{$R T$-PCR for HSV-TK expression}

TK expression in stably transduced DHD/K12 clones and in tumor nodes was investigated by RT-PCR. Tumor nodes were harvested from animals freshly dead from extensive peritoneal dissemination. Total cellular RNA was extracted by Tripure reagent (Boehringer Mannheim). One microgram of RNA was first reverse transcribed (MoMLV reverse transcriptase, GIBCO BRL) with a specific $T K-3^{\prime}$ primer (5'-ACAGGGTAAATAACGTGTC-3'). The cDNA product was then amplified with the TK primers 5'-ATGGCTTCGTACCCCTG-3' and 5'-AAGGTCGGCGGGATGAG-3'. The amplification was performed for 35 cycles (denaturation at $95^{\circ} \mathrm{C}$ for $1 \mathrm{~min}$, annealing at $50^{\circ} \mathrm{C}$ for $1 \mathrm{~min}$ and elongation at $72^{\circ} \mathrm{C}$ for 2 min) followed by a 4 -min final elongation at $72^{\circ} \mathrm{C}$. The RT-PCR products were transferred to a nylon membrane (Quiabrane; Qiagen, Hilden, Germany) and analyzed by Southern blotting. The TK-DNA probe was the ${ }^{32} \mathrm{P}$ random labeled RT-PCR product from stably transfected DHD/K12-TK cells.

\section{Animal treatment}

BD-IX rats were injected intraperitoneally at day 0 with $10^{6}$ cells and then treated by intraperitoneal injections of GCV diluted in Hank's balanced salt solution (HBSS; GIBCO BRL) or injections of the HBSS buffer alone for 5 days starting at day 14 . In the experiment described in Figure 4, the animals were killed at day 21 and the tumor dissemination was evaluated by direct abdominal examination. In the other experiments, animals were allowed to die from extensive peritoneal disease and survival curves were drawn.

In the experiment described in Figure 7, 5-day GCV treatments (150 mg/kg once daily) were repeated every 4 weeks.

For the peritoneal irritations, the animals were laparotomized and a dissection of the sigmoid was performed without any intestinal resection.

\section{Statistics}

Kaplan-Meier curves were established for each group and survivals were compared with the log-rank test.

\section{Acknowledgements}

We thank C Grignet and Dr C-M Calberg for the retroviral vector and the TK-producing GP+EnvAm12 packaging cell line. We are most thankful to Prof N Jacquet for his support and to Mrs Rocoux for her excellent technical assistance. We thank W Dewez for his help for statistical analysis. CL is supported by a Télévie fellowship and FP by an FRIA fellowship. VB and M-PM are Research Associates at the National Fund for Scientific Research (Belgium). This research was supported by grants from Télévie and the 'Centre Anti-Cancéreux' (Liège, Belgium). 


\section{References}

1 Markman M. Intraperitoneal chemotherapy. Semin Oncol 1991; 18: 248-254.

2 Schneider JG. Intraperitoneal chemotherapy. Obst Gynecol Clin North Am 1994; 21: 195-212.

3 Freeman SM et al. In situ use of suicide genes for cancer therapy. Semin Oncol 1996; 23: 31-45.

4 Field AK et al. 9-2-Hydroxy-1-(hydromethyl)ethoxy-methylguanine: a selective inhibitor of herpes group virus replication. Proc Natl Acad Sci USA 1983; 80: 4139-4143.

5 Davidson RL, Kaufman ER, Crumpacker CS, Schnipper LE. Inhibition of herpes simplex virus transformed cells by acycloguanosine: mechanisms of uptake and toxicity. Virology 1981; 113: 9-19.

6 Elion GB et al. Selectivity of action of an antiherpetic agent, 9(2-hydroxyethoxymethyl)guanine. Proc Natl Acad Sci USA 1977; 74: 5716-5720.

7 Moolten FL. Tumor chemosensitivity conferred by inserted herpes thymidine kinase genes: paradigm for a prospective cancer control strategy. Cancer Res 1986; 46: 5276-5281.

8 Moolten FL, Wells JM. Curability of tumors bearing herpes thymidine kinase genes transferred by retroviral vectors. J Natl Cancer Inst 1990; 82: 297-300.

9 Moolten FL, Vonderhaar BK, Mroz PJ. Transduction of the herpes thymidine kinase gene into premalignant murine mammary epithelial cells renders subsequent breast cancers responsive to ganciclovir therapy. Hum Gene Ther 1996; 7: 1197-1204.

10 Barba D, Hardin J, Ray J, Gage FH. Thymidine kinase-mediated killing of rat brain tumors. J Neurosurg 1993; 79: 729-735.

11 Vile RG et al. Systemic gene therapy of murine melanoma using tissue specific expression of the HSV $t k$ gene involves an immune system. Cancer Res 1994; 54: 6228-6234.

12 Freeman SM et al. The 'bystander-effect': tumor regression when a fraction of the tumor mass is genetically modified. Cancer Res 1993; 53: 5274-5283.

13 Smythe WR et al. Use of recombinant adenovirus to transfer the herpes simplex virus thymidine kinase (HSVtk) gene to thoracic neoplasms: an effective in vitro drug sensitization system. Cancer Res 1994; 54: 2055-2059.

14 Caruso $\mathrm{M}$ et al. Regression of established macroscopic liver metastases after in situ transduction of a suicide gene. Proc Natl Acad Sci USA 1993; 90: 7024-7028.

15 Culver KW et al. In vivo gene transfer with retroviral vector- producer cells for treatment of experimental brain tumors. Science 1992; 256: 1550-1552.

16 Chen S-H et al. Gene therapy for brain tumors: regression of experimental gliomas by adenovirus-mediated gene transfer in vivo. Proc Natl Acad Sci USA 1994; 91: 3054-3057.

17 Ram $\mathrm{Z}$ et al. In situ retroviral-mediated gene transfer for treatment of brain tumors in rats. Cancer Res 1993; 53: 83-88.

18 O'Malley BW, Chen S-H, Schwartz MR, Woo SLC. Adenovirusmediated gene therapy for human head and neck squamous cell cancer in a nude mouse model. Cancer Res 1995; 55: 1080-1085.

19 Vile RG, Hart IR. Use of tissue-specific expression of the herpes simplex virus thymidine kinase gene to inhibit growth of established murine melanomas following direct intratumoral injection of DNA. Cancer Res 1993; 53: 3860-3864.

20 Aoki K, Yoshida T, Sugimura T, Terada M. Liposome-mediated in vivo gene transfer of antisense $\mathrm{K}$-ras construct inhibits pancreatic tumor dissemination in the murine peritoneal cavity. Cancer Res 1995; 55: 3810-3816.

21 Smythe WR et al. Successful adenovirus-mediated gene transfer in an in vivo model of human malignant mesothelioma. Ann Thorac Surg 1994; 57: 1395-1401.

22 Smythe WR et al. Treatment of experimental human mesothelioma using adenovirus transfer of the herpes simplex thymidine kinase gene. Ann Surg 1995; 222: 78-86.

23 Bi WL, Parysek LM, Warnick R, Stambrook PJ. In vitro evidence that metabolic cooperation is responsible for the bystander effect observed with HSV TK retroviral gene therapy. Hum Gene Ther 1993; 4: 725-731.

24 Colombo BM et al. The 'bystander effect': association of U-87 cell death with ganciclovir-mediated apoptosis of nearby cells and lack of effect in athymic mice. Hum Gene Ther 1995; 6: 763-772.

25 Samejima Y, Meruelo D. 'Bystander killing' induces apoptosis and is inhibited by forskolin. Gene Therapy 1995; 2: 50-58.

26 Daerden-Badet M-T, Pelletier H, Caignard A, Martin F. In vitro proliferative responses of spleen lymphocytes from rats bearing progressive or regressive tumors induced by cell variants of a syngeneic colon carcinoma. Int J Cancer 1989; 43: 334-339.

27 Martin F et al. Selection by trypsin of two sublines of rat colon cancer cells forming progressive or regressive tumors. Int $J$ Cancer 1983; 32: 623-627.

28 Markowitz D, Goff S, Bank A. Construction and use of a safe and efficient amphotropic packaging cell line. Virology 1988; 167: 400-406. 